\title{
YAP is overexpressed in clear cell renal cell carcinoma and its knockdown reduces cell proliferation and induces cell cycle arrest and apoptosis
}

\author{
JIAN-JIA CAO ${ }^{1}$, XIU-MIN ZHAO $^{1}$, DE-LIN WANG ${ }^{1}$, KE-HONG CHEN $^{1}$, XIA SHENG $^{1}$, \\ WEN-BIN LI ${ }^{1}$, MEI-CAI LI ${ }^{1}$, WU-JIANG LIU ${ }^{2}$ and JIANG HE ${ }^{3}$ \\ ${ }^{1}$ Department of Urology, The First Affiliated Hospital of Chongqing Medical University, Chongqing; \\ ${ }^{2}$ Institute of Urology, First Hospital of Peking University, Beijing; ${ }^{3}$ Gastroenterology and Neurology Center, \\ University-Town Hospital of Chongqing Medical University, Chongqing, P.R. China
}

Received May 6, 2014; Accepted June 23, 2014

DOI: 10.3892/or.2014.3349

\begin{abstract}
Yes-associated protein (YAP) has been reported to be an oncogene in a number of malignancies. It constitutes an important regulatory mechanism for the Hippo pathway, a key regulator of cell growth and apoptosis. The present study aimed to investigate the clinical significance and the role of YAP in the development of clear cell renal cell carcinoma (ccRCC). YAP expression levels were compared between ccRCC and adjacent normal renal tissues by RT-PCR and immunohistochemistry, respectively. YAP expression levels were then detected in ccRCC cell lines 786-0 and ACHN, as well as in human embryonic kidney 293 cells (HEK-293) using western blotting. Three specific YAP-shRNA lentiviral vectors were constructed and transfected into 786-0 cells, and then the mRNA and protein levels of YAP and downstream transcription factor TEAD1 were detected. Finally, the effects of YAP silencing on proliferation and the cell cycle distribution of 786-0 cells were detected by Cell Counting Kit-8 (CCK-8) and flow cytometry (FCM), respectively. The apoptosis rate was also analyzed by FCM. It was observed that the expression levels of YAP mRNA and protein in ccRCC tissues were higher than these levels in the adjacent normal renal tissues. The expression of YAP protein in ccRCC tissues was significantly correlated with clinical stage and differentiation. The YAP protein levels in the two ccRCC cell lines 786-0 and ACHN were significantly higher than that in the HEK-293 cells. Additionally, treatment of 786-0 cells with YAP-shRNA lentiviral vectors significantly reduced the expression levels of YAP and TEAD1 mRNA and protein. Further analyses in 786-0 cells in which YAP was decreased, revealed that cell
\end{abstract}

Correspondence to: Professor De-Lin Wang, Department of Urology, The First Affiliated Hospital of Chongqing Medical University, 1 Youyi Road, Yuzhong, Chongqing 400016, P.R. China

E-mail: dlwangws@sina.com

Key words: clear cell renal cell carcinoma, Yes-associated protein, TEAD1, RNA interference, lentivirus proliferation was inhibited, cell cycle was arrested at the G1 phase and apoptosis was increased. These results indicate that YAP is an underlying oncogene in ccRCC and it may be a promising biomarker and therapeutic target of ccRCC.

\section{Introduction}

As one of the most common malignant tumors in the urinary system, renal cancer accounts for more than 270,000 new cases worldwide each year (1). Clear cell renal cell carcinoma (ccRCC) is the most common histological subtype, accounting for $75-85 \%$ of all RCC cases. The incidence of RCC has steadily increased in many countries (2). However, the treatment options for RCC are limited, and the mechanisms of the pathogenesis of renal carcinoma are not completely defined. Therefore, new treatment strategies for patients with RCC are urgently needed. With continued research on the mechanisms of tumors, targeted therapy has made remarkable progress (3). In recent years, novel markers and therapeutic targets of RCC have continued to emerge. Nevertheless, to date, no biomarkers have been used in RCC in the routine clinic (4).

The Hippo signaling pathway, initially discovered in Drosophila, is a potent regulator of cell growth and apoptosis (5). A series of biochemical and genetic studies identified this pathway whose components are highly conserved between Drosophila and mammals $(6,7)$. In mammals, the components of the Hippo pathway include MST1/2, Sav1, LATS1/2, YAP, TAZ and TEAD $(8,9)$. MST1/2, Sav1 and LATS1/2 are regarded as upstream kinases of the mammalian Hippo pathway. It is presumed that through phosphorylation of YAP, the upstream kinases LATS1/2 inhibit YAP transcription activity (10). YAP, TAZ and TEAD were identified as downstream transcription factors of this pathway (11).

Yes-associated protein (YAP), the mammalian homolog of Drosophila Yorkie (Yki), is a $65-\mathrm{kD}$ a protein which was originally identified due to its interaction with the Src family tyrosine kinase c-Yes (12). Recent studies have found that the transcriptional co-activator YAP is a key nuclear effector of the Hippo pathway which regulates organ size by governing cell proliferation and apoptosis $(13,14)$. The TEAD family, 
located in the downstream of the Hippo pathway, are major mediators of YAP transcriptional activity $(15,16)$. The domain of YAP which interacts with the TEAD family of transcription factors is essential for YAP-mediated tumor growth and metastasis (17). Recently, YAP has been shown to be a candidate oncogene in recurrent amplification at human chromosome 11q22 (18). Overexpression of YAP protein and its nuclear localization were observed in colon, lung, pancreatic, hepatocellular, ovarian and prostate carcinomas $(16,19,20)$.

Despite of the growing evidence of YAP as a crucial regulator of human types of cancers, its involvement in ccRCC is still obscure. In the present study, we attempted to identify the role of YAP in ccRCC. We evaluated the expression levels and clinical significance of YAP in ccRCC tissues. In addition, we investigated the effects of YAP silencing via YAP-shRNA lentiviral vectors transfected into ccRCC cell lines. These findings suggest that YAP may serve as a potential target in the treatment of ccRCC.

\section{Materials and methods}

Tissue samples and cell culture. Tissue samples from radical nephrectomy were obtained from the First Affiliated Hospital of Chongqing Medical University from March 2010 to November 2011. The tissue samples consisted of 30 cases of ccRCC tissues and 30 cases of adjacent normal renal tissues (4 $\mathrm{cm}$ or more from the tumor). Samples were confirmed by a pathologist and stored at $-80^{\circ} \mathrm{C}$. The present study was conducted with approval from the Ethics Committee of the First Affiliated Hospital of Chongqing Medical University. Informed consent was obtained from all patients. Human ccRCC cell lines 786-0 and ACHN and the human HEK-293 cell line were purchased from ATCC. ACHN and 786-0 cells were cultured in RPMI-1640 medium, and HEK-293 in DMEM. All medium contained $10 \%$ fetal bovine serum (FBS) (Gibco-BRL, Carlsbad, CA, USA). All cells were maintained in an incubator with $5 \% \mathrm{CO}_{2}$ at $37^{\circ} \mathrm{C}$.

Immunohistochemistry. The tissues were fixed with $10 \%$ formaldehyde (ZSGB-Bio, Beijing, China) embedded in paraffin, sliced into $4-\mu \mathrm{m}$ sections and used for staining. In brief, the sections were rehydrated, and antigen retrieval was performed by a microwave vacuum histoprocessor (RHS-1; Bergamo, Italy) at $121^{\circ} \mathrm{C}$ for $15 \mathrm{~min}$. After being blocked with goat serum (Gibco-BRL), the primary antibody against YAP (Santa Cruz, Dallas, TX, USA) was diluted (1:200) and incubated with the tissue sections overnight in a humidity chamber at $4^{\circ} \mathrm{C}$. The sections were treated with horseradish peroxidase (HRP)-labeled secondary antibody (ZSGB-Bio) and DAB, and the results were observed with a microscope. The tissues were scored according to the percentage of the positively stained area and staining intensity. The percentage of the positive area was graded as $0(\leq 5 \%), 1(6-25 \%), 2(26-50 \%), 3(\geq 51 \%)$, and the staining intensity was graded as 0-2 (i.e., negative, 0 ; weak, 1 and strong, 2). The two grades were multiplied and tissues were assigned to one of 3 levels: 0 , negative; $1-4$, weak positive; and 5-6, strong positive.

Construction of the lentiviral interference vectors and transfection. According to the YAP gene sequence (NM_006106) in the GenBank, three sequences of the shRNA targeting YAP were designed as follows: 5'-GCTCATTCCTCTCCAGCTT-3', 5'-CCTTAACAGTGGCACCTAT-3' and 5'-CCGTTTCCCAG ACTACCTT-3'. Non-silencing shRNA (5'-TTCTCCGAAC GTGTCACGT-3') was synthesized as the negative control (NC shRNA). The YAP lentiviral interference vector was designed and constructed by Shanghai SBO Medical Biotechnology. Briefly, for generation of shRNA-YAP or control lentiviral vector, the lentivirus expressing plasmids pLTR-G and pNLEGFP/CMV/WPREdU3 which were carrying shRNA, along with lentivirus packing plasmids $\mathrm{pCD} / \mathrm{NL}-\mathrm{BH}^{*} \mathrm{DDD}$ (both from Addgene, USA) were added into 293T cells for lentiviral vector packaging. Eventually, the lentivirus was extracted and lentivirus concentrations were verified. For lentivirus transfection, 786-0 cells were grown to $30-50 \%$ confluency and transfected with three pairs of YAP-shRNAs and the nonsilencing control shRNA at an optimized multiplicity of infection (MOI) of 20 with Polybrene at a concentration of $5 \mu \mathrm{g} / \mathrm{ml}$. The successful transfection of YAP-shRNA lentiviral vectors was observed by fluorescence microscope $96 \mathrm{~h}$ after transfected. The YAP-shRNA infected cells were detected as GFP-positive cells under fluorescence microscopy at a magnification of $\mathrm{x} 200$ (Olympus, Tokyo, Japan). To detect the transfection efficiency, the mRNA and protein levels of YAP were respectively assessed by RT-PCR and western blotting $96 \mathrm{~h}$ after transfection.

RT-PCR. The tissues and the cultured cells were collected. Total RNA were extracted using the TRIzol kit (Takara, Osaka, Japan). The total RNA quality was detected by a UV spectrophotometer (Bio-Rad, Hercules, CA, USA), and $1 \mu \mathrm{g}$ total RNA was transcribed reversely for the cDNA and PCR reaction amplification of YAP and TEAD1 genes, and GAPDH as an internal reference. Specific primers were designed: YAP-F, 5-TGAACAAACGTCCAGCAAGATAC-3 and YAP-R, 5-CAGCCCCCAAAATGAACAGTAG-3; TEAD1-F, 5-TGAATCAGTGGACATTCGTCA-3 and TEAD1-R, 5-GC CATTCTCAAACCTTGCATA-3; and GAPDH-F, 5-ACCAC CATGGAGAAGGCTGG-3 and GAPDH-R, 5-CTCAGTTAG CCCAGGATGC-3. PCR products were subjected to agarose gel electrophoresis (SABC, China), UV light observation and photography. A Bio-Rad gel formatter was used to analyze the original band.

Western blotting. The YAP antibody was purchased from Santa Cruz Biotechnology (Santa Cruz, CA, USA) and the TEAD1 antibody was purchased from Proteintech Group, Inc. (Chicago, IL, USA). Total protein was extracted from the tissue homogenates and the cell lysate by RIPA buffer (50 mM Tris, pH 7.4, $150 \mathrm{mM} \mathrm{NaCl,} \mathrm{1 \%} \mathrm{Triton} \mathrm{X-100,} \mathrm{1 \%}$ Nonidet-P40, $0.5 \%$ sodium deoxycholate, $0.1 \%$ SDS, $1 \mathrm{mM}$ EDTA and PMSF) (Beyotime, Shanghai, China). The lysate containing $50 \mu \mathrm{g}$ protein quantified by the BCA protein assay kit for each sample was subjected to SDS-PAGE, and was then transferred to a $0.45-\mu \mathrm{m}$ polyvinylidene fluoride (PVDF) membrane (both from Beyotime). The membrane was blocked in Tris-buffered saline-Tween $0.1 \%$ (TBST) with $5 \%$ skimmed milk powder for $2 \mathrm{~h}$ at $37^{\circ} \mathrm{C}$. The primary antibodies were diluted (YAP 1:200, TEAD1 1:200 and $\beta$-actin 1:1,000). The proper species and diluted HRP-labeled 


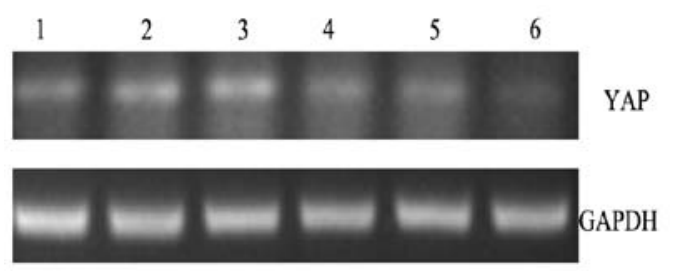

Figure 1. Expression of YAP mRNA and GAPDH mRNA by RT-PCR. (Lanes 1-3, ccRCC tissues; lanes 4-6, normal renal tissues). Total RNA from 30 cases of ccRCC and 30 cases of adjacent normal renal tissues was extracted. cDNA and specific primers were synthesized. PCR products were electrophoresed on a $2.5 \%$ agarose gel and analyzed by Bio-Rad gel formatter. Expression of GAPDH is shown for comparison. YAP, Yes-associated protein; ccRCC, clear cell renal cell carcinoma.

secondary antibodies were added, and Western blot results were analyzed by Bio-Rad software.

Proliferation assay. The 786-0 cells were seeded in 96-well plates for $12 \mathrm{~h}$ at a concentration of $5 \times 10^{3}$ cells/well. Cells were transfected with shYAP-3 or NC shRNA lentiviral vectors. After treatment for 48, 96 and $144 \mathrm{~h}, 10 \mu \mathrm{l}$ of Cell Counting Kit-8 (CCK-8) reagent (Sigma, USA) in $90 \mu 1$ RPMI-1640/well was added to the cell culture medium and then incubated at $37^{\circ} \mathrm{C}$ for $1 \mathrm{~h}$. The absorbance was measured with a microplate reader (Spectramax M2; MD, USA) at $450 \mathrm{~nm}$.

Cell cycle assay by flow cytometry (FCM). The 786-0 cells were transfected with the shYAP-3 lentiviral vector which was the most efficient inhibition transfected group or NC shRNA for $96 \mathrm{~h}$, and then harvested abundantly. The cells were washed with PBS twice, and fixed with $70 \%$ ethanol overnight at $4^{\circ} \mathrm{C}$. Fixed cells were stained with $50 \mu \mathrm{g} / \mu \mathrm{l}$ propidium iodide (PI) (Bioscience, Shanghai, China) and $100 \mu \mathrm{g} / \mu 1 \mathrm{RNase}$ (Sigma). Cell cycle distribution of each group was measured with a flow cytometer (Beckman Coulter, USA).

Apoptosis assay. The 786-0 cells were transfected with shYAP-3 or the NC shRNA lentiviral vector for $96 \mathrm{~h}$, then trypsinized and washed with PBS, resuspended in $100 \mu \mathrm{l}$ binding buffer, and stained with 7-aminoactinomycin D (7-AAD) and PE-conjugated Annexin V (Bioscience). The apoptosis rate of each group was measured with a flow cytometer (Beckman Coulter).

Statistical analysis. Statistical analysis was performed with SPSS 19.0 software. Data are presented as the means \pm standard deviation. The Chi-square trend test was used to assess the correlation between YAP protein expression and clinicopathologic factors of the renal cancer cases. A Student's two-tailed t-test was used to compare two groups. One-way ANOVA was used in comparison of means among multiple groups. $p<0.05$ was considered to indicate a statistically significant result.

\section{Results}

Expression of YAP in ccRCC tissues and correlation with clinicopathological factors. As determined by RT-PCR, the expression level of YAP mRNA in the ccRCC tissues was $0.569 \pm 0.066$, while that in adjacent normal renal tissues was
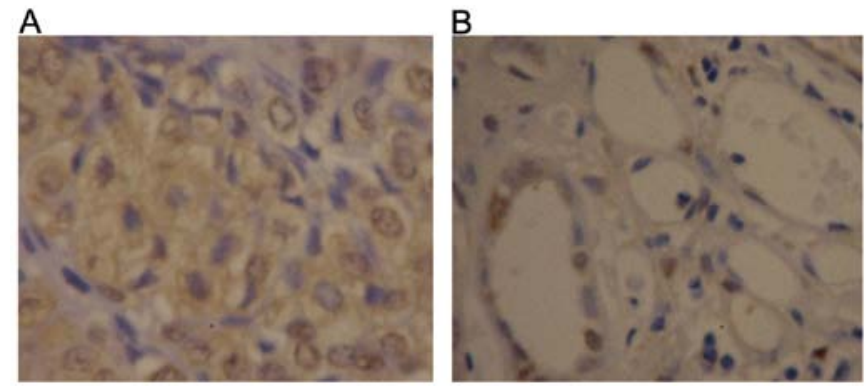

Figure 2. Expression of YAP protein as detected by immunohistochemistry (magnification, $\mathrm{x} 400$ ). (A) Low expression of YAP protein in adjacent normal renal tissues; (B) high expression of YAP protein in ccRCC tissues. YAP, Yes-associated protein; ccRCC, clear cell renal cell carcinoma.

Table I. Association of YAP protein expression with clinicopathological factors of the ccRCC cases $\left(\chi^{2}\right.$-test).

\begin{tabular}{|c|c|c|c|c|c|}
\hline \multirow[b]{2}{*}{ Factors } & \multirow[b]{2}{*}{ Patients } & \multicolumn{2}{|c|}{$\begin{array}{c}\text { YAP } \\
\text { expression }\end{array}$} & \multirow{2}{*}{$\begin{array}{l}\text { YAP-positive } \\
\text { rate }(\%)\end{array}$} & \multirow[b]{2}{*}{ P-value } \\
\hline & & $(+)$ & $(-)$ & & \\
\hline Gender & & & & & 0.705 \\
\hline Male & 15 & 10 & 5 & 66.7 & \\
\hline Female & 15 & 9 & 6 & 60.0 & \\
\hline Age (years) & & & & & 0.757 \\
\hline$<60$ & 18 & 11 & 7 & 61.1 & \\
\hline$\geq 60$ & 12 & 8 & 4 & 66.7 & \\
\hline $\begin{array}{l}\text { Renal vein } \\
\text { metastasis }\end{array}$ & & & & & 0.265 \\
\hline Present & 2 & 2 & 0 & 100.0 & \\
\hline None & 28 & 17 & 11 & 60.7 & \\
\hline Tumor size $(\mathrm{cm})$ & & & & & 0.424 \\
\hline$>5$ & 8 & 6 & 2 & 75.0 & \\
\hline$\leq 5$ & 22 & 13 & 9 & 59.1 & \\
\hline Differentiation & & & & & 0.018 \\
\hline Well & 8 & 3 & 5 & 33.3 & \\
\hline Moderate & 13 & 8 & 5 & 61.5 & \\
\hline Poor & 9 & 8 & 1 & 88.9 & \\
\hline Clinical stage & & & & & 0.034 \\
\hline I-II & 17 & 8 & 9 & 47.1 & \\
\hline III-IV & 13 & 11 & 2 & 84.6 & \\
\hline
\end{tabular}

YAP, Yes-associated protein; ccRCC, clear cell renal cell carcinoma.

$0.515 \pm 0.068$. The expression level of YAP mRNA in ccRCC tissues was significantly higher than that in adjacent normal renal tissues $(\mathrm{p}<0.05)$ (Fig. 1).

The expression of YAP protein in 30 cases of ccRCC tissues and 30 cases of adjacent normal renal tissues was detected by immunohistochemistry. In the ccRCC tissues, 63.3\% (19/30) of the samples exhibited positive expression, and the positive areas were primarily located in the nucleus and cytoplasm (Fig. 2A). In contrast, the positive rate of YAP protein expression in the normal tissues was $33.3 \%$ (10/30); most cases showed weak 
A
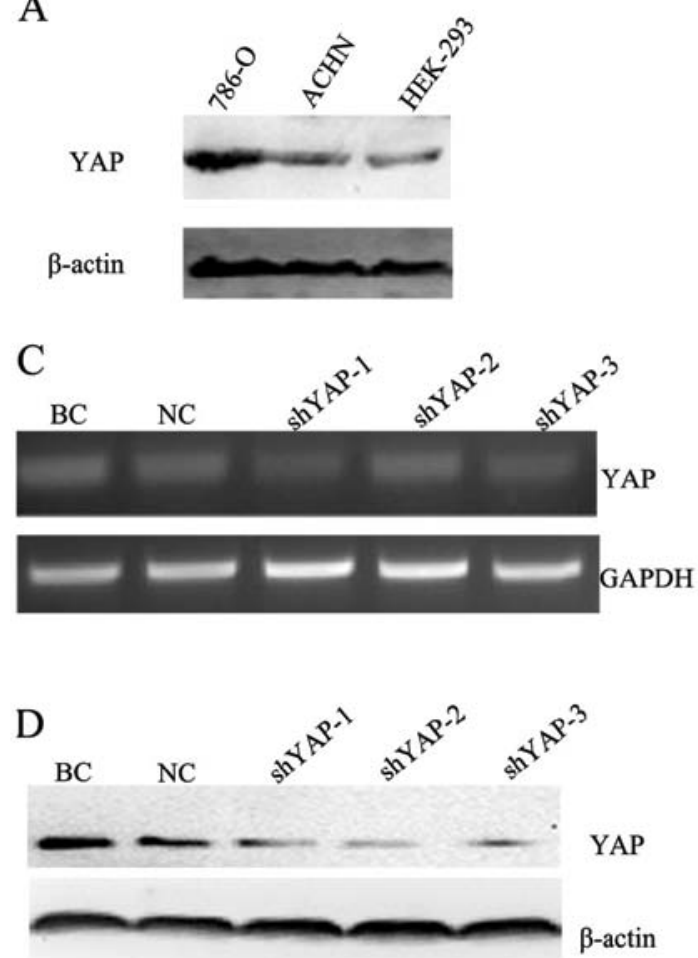

B

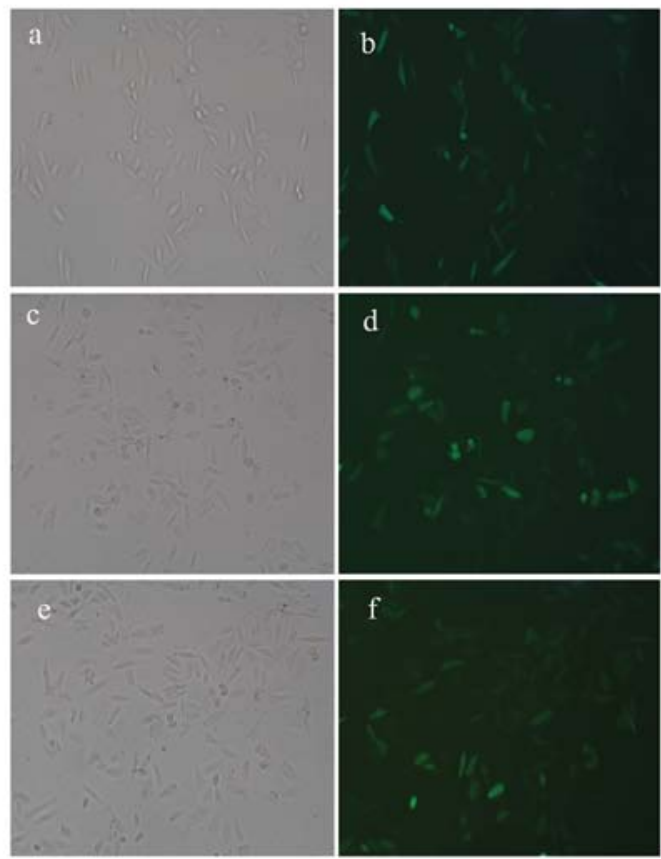

Figure 3. YAP-shRNA lentiviral vector was transfected into 786-0 cells and suppressed the expression levels of YAP mRNA and protein. (A) The YAP protein levels in two ccRCC cell lines and HEK-293 cells. (B) GFP expression in 786-0 cells at $96 \mathrm{~h}$ after transfection with the YAP-shRNA lentiviral vector as shown by fluorescence microscopy (magnification, x200). a, c and e images, respectively, represent shYAP-1, the shYAP-2 and shYAP-3 transfected group cells under light microscopy; b, d and f images, respectively, represent shYAP-1, shYAP-2 and shYAP-3 transfected group cells under fluorescence microscopy. (C) The 786-0 cells were transfected with shYAP-1, shYAP-2, shYAP-3 and NC lentiviral vector, and the BC group was used as a blank control. YAP mRNA expression was assessed by RT-PCR. Expression of GAPDH is shown for comparison. (D) Expression of YAP protein in the different groups was confirmed by western blotting. Expression of $\beta$-actin is shown for comparison. BC, blank control group, cells only; NC, negative control group, negative control vector; YAP, Yes-associated protein; ccRCC, clear cell renal cell carcinoma.

A

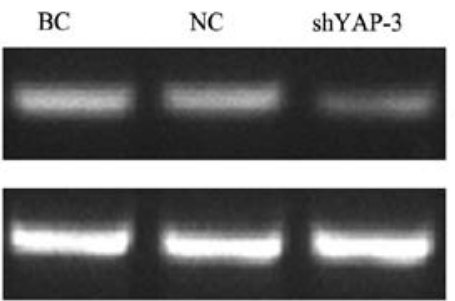

B

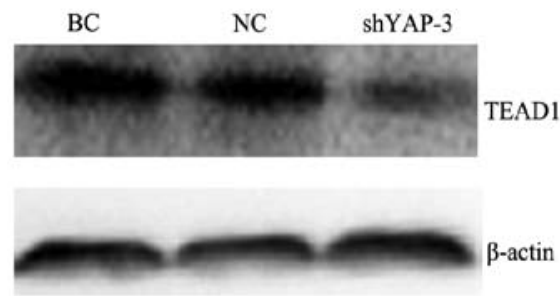

Figure 4. The suppressive effect of TEAD1 mRNA and protein in 786-0 cells after shYAP-3 lentiviral vector transfection. (A) The expression of TEAD1 mRNA in different groups by RT-PCR. (B) The expression of TEAD1 protein in the different groups by western blotting. The shYAP-3-transfected group showed reduced TEAD1 mRNA and protein expression $(\mathrm{p}<0.05)$, while the TEAD1 mRNA and protein were not significantly different between the blank control and the negative control group $(\mathrm{p}=0.864)$. $\mathrm{BC}$, blank control group, cells only; $\mathrm{NC}$, negative control group, negative control vector.

positive expression and the YAP protein was confined to the renal collecting duct system (Fig. 2B). The positive rate of YAP protein in the ccRCC tissues was obviously higher than that in the adjacent normal renal tissues $(p<0.05)$.

We further analyzed the correlation of YAP protein expression with clinicopathological factors of ccRCC. As shown in Table I, there was no obvious relationship between YAP protein expression and gender, age, renal vein metastasis or tumor size. However, the positive rate of YAP protein expression was $33.3 \%(3 / 8)$ in well differentiated, $61.5 \%(8 / 13)$ in moderately differentiated and $88.9 \%$ (8/9) in low differentiated tumors $(\mathrm{p}=0.018)$. In addition, the positive rate of YAP protein expression was $47.1 \%$ (8/17) in stage I-II and $84.6 \%$
(11/13) in stage III-IV cases ( $\mathrm{p}=0.034)$. These results showed that the expression level of YAP protein was closely correlated to clinical stage and differentiation in the ccRCC tissues.

Expression of YAP in 786-O cells is suppressed by shRNA lentiviral vectors. We examined the expression level of YAP protein in ccRCC cell lines 786-0 and ACHN and in the human embryonic kidney HEK-293 cells using western blotting. As shown in Fig. 3A, the YAP protein levels in the two ccRCC cell lines 786-0 and ACHN were significantly higher than that in the HEK-293 cells $(\mathrm{p}<0.05)$.

To determine whether YAP plays a role in tumorigenesis of renal cancer, three specific YAP-shRNA lentiviral vectors, 


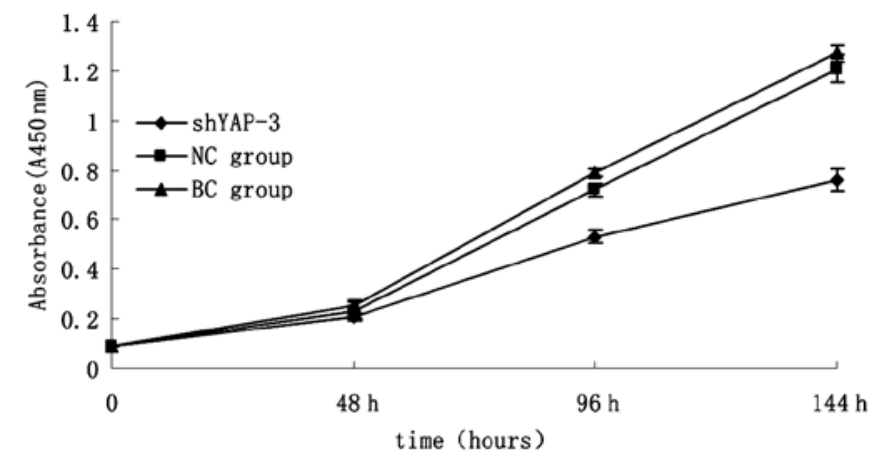

Figure 5. Effect of YAP silencing on the proliferation of 786-0 cells. The absorbance values in the shYAP-3-transfected group, the blank control group and negative control group cells were measured at 48, 96 and $144 \mathrm{~h}$, respectively. The absorbance values represent the mean from three independent experiments. YAP, Yes-associated protein.

shYAP-1, shYAP-2 and shYAP-3, were successfully constructed and transfected into 786-0 cells. After $96 \mathrm{~h}$ of transfection, as shown in Fig. 3B, 786-0 cells were successfully transfected with the YAP-shRNA lentiviral vectors. Three YAP interference groups, as well as a blank control group (BC group, cells only) and negative control group (NC group) were established from the 786-0 cells. Furthermore, the efficient knockdown of YAP expression was detected by RT-PCR and Western blot analysis. RT-PCR analysis showed that the relative mRNA expression level of YAP in the YAP-shRNA transfection cells was markedly lower than levels in the $\mathrm{BC}$ and $\mathrm{NC}$ groups $(\mathrm{p}<0.05)$ (Fig. 3C). Similarly, the expression of YAP protein was also significantly inhibited as confirmed by western blotting ( $\mathrm{p}<0.05$ ) (Fig. 3D). The lowest level of YAP expression was observed in the cells transfected with the shYAP-3 lentiviral vectors. These results indicated that the shYAP-3 lentiviral vector had the highest inhibition rate of the three specific
YAP-shRNA lentiviral vectors. Therefore, the shYAP-3 lentiviral vector was chosen for subsequent experiments.

Knockdown of YAP inhibits expression of TEAD1. RT-PCR analysis showed that the relative mRNA expression level of TEAD1 in the shYAP-3-transfected cells was markedly lower than levels in the $\mathrm{BC}$ and $\mathrm{NC}$ group cells $(\mathrm{p}<0.05)$ (Fig. 4A). As shown in the western blot analysis (Fig. 4B), we also observed that the protein expression level of TEAD1 was significantly decreased compared with the levels in the BC and NC groups $(\mathrm{p}<0.05)$, indicating that silencing of YAP inhibited the expression level of TEAD1, which is a downstream gene in the Hippo pathway.

YAP knockdown suppresses cell proliferation. To determine whether the YAP-shRNA lentiviral vector actually affects the proliferation of 786-0 cells, we examined the proliferation of the three 786-0 cell groups including the shYAP-3-transfected group, the blank group and negative control group. As shown in Fig. 5, after silencing of YAP in the 786-0 cells for $144 \mathrm{~h}$, we found that there was a significant reduction in cell proliferation $(\mathrm{p}<0.05)$. In addition, the NC group presented a minimal effect on the proliferation of 786-0 cells compared with the blank control group ( $p>0.05$ ). The results demonstrated that YAP plays an important role in cell survival, and the knockdown of the YAP gene leads to suppression of renal cancer cell proliferation.

YAP knockdown arrests the cell cycle and induces cell apoptosis. Ninety-six hours after shYAP-3 lentiviral vector transfection, the effect of YAP on the cell cycle progression was analyzed by FCM. In the shYAP-3-transfected group, we found that $61.4 \%$ of cells were in the G1 phase and $29.4 \%$ were in the $\mathrm{S}$ phase, compared with $51.4 \%$ of cells in the G1 phase and $39.4 \%$ in the $\mathrm{S}$ phase in the blank control group and $51.0 \%$
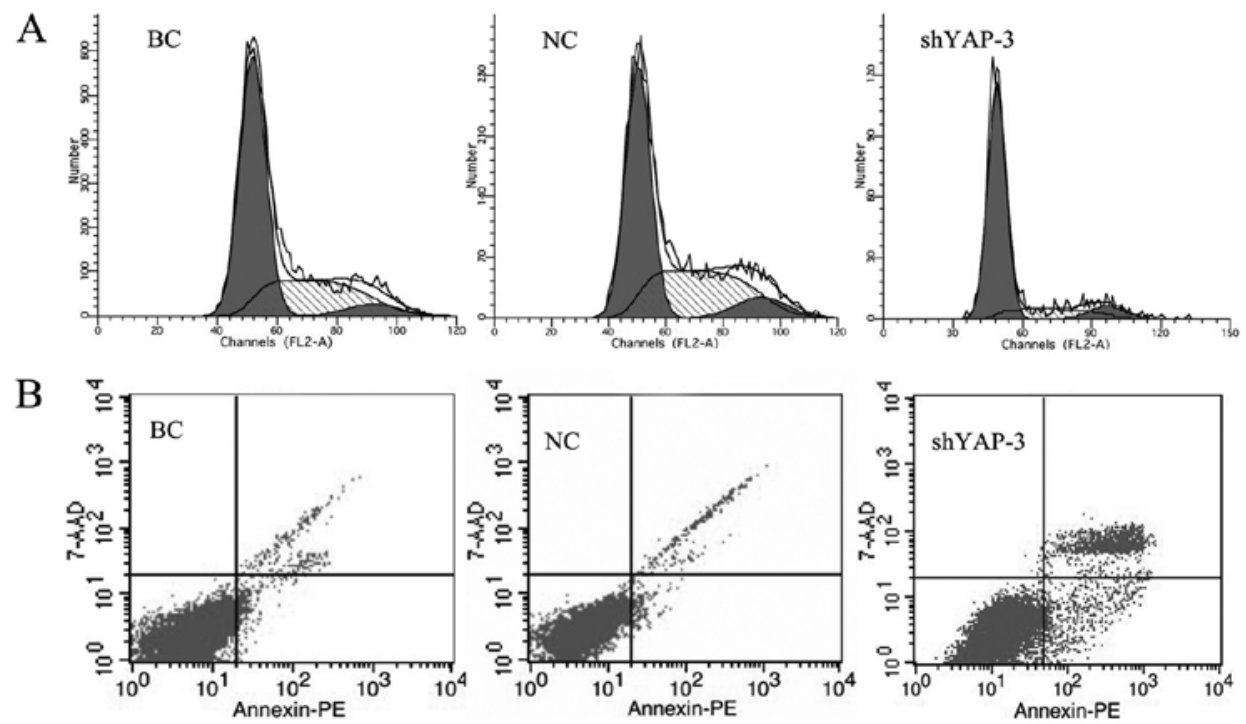

Figure 6. Effect of YAP silencing on cell cycle progression and the apoptosis rate in 786-0 cells. (A) Flow cytometric analysis of the cell cycle of 786-0 cells transfected with shYAP-3 lentiviral vectors or the control group after $96 \mathrm{~h}$. The cell cycle of the shYAP-3 transfected group was compared with the control group and the proportion of cells in the G1 phase was significantly increased, while the proportion of cells in the $\mathrm{S}$ phase was significantly reduced ( $\mathrm{p}<0.05)$. (B) The apoptosis rate of the shYAP-3-transfected group cells was significantly higher than the rates in the BC and NC group cells ( $<<0.05$ ). YAP, Yes-associated protein; BC, blank control group; NC, negative control group; shYAP-3, shYAP-3-transfected group. 
of cells in G1 phase and $39.8 \%$ in S phase in the NC group $(\mathrm{p}<0.05)$ (Fig. 6A). No significant difference was observed in cell cycle progression between the blank control and the NC group ( $p>0.05$ ). Furthermore, the apoptosis rate of cells with YAP knockdown was analyzed by FACS. The results showed that the apoptosis rate in the 786-0 cells with YAP knockdown was significantly increased as compared with the rates in the control groups $(\mathrm{p}<0.05)$ (Fig. 6B). The difference between the blank control group and the NC group was not obvious $(\mathrm{p}=0.582)$. This finding suggests that knockdown of YAP induced 786-0 cell apoptosis and G1 phase arrest.

\section{Discussion}

The Hippo pathway has been recognized as an important regulator of both organ size control and tumorigenesis (6). In mammals, the Hippo pathway negatively regulates cell growth through a kinase cascade and results in the inactivation of YAP. Hippo core components form protein kinase complexes acting in a cascade to phosphorylate YAP and induce its cytoplasmic translocation (21). YAP expression has been significantly associated with tumor metastasis, grade and stage (22). A study in lung cancer showed that YAP expression was significantly correlated with TNM stage (23).

In the present study, YAP mRNA and protein expression levels in 30 cases of ccRCC tissues and 30 cases of adjacent normal renal tissues were first detected. The results indicated that expression levels of YAP mRNA and protein in ccRCC tissues were significantly higher than those in adjacent normal renal tissues. ccRCC tissues had a high frequency of YAP-positive cells, whereas the adjacent normal tissues had a lower frequency $(63.3 \%$ vs. $33.3 \%)$. Although a significant percentage of adjacent normal cases stained positive for YAP, the overall expression level was significantly higher in the tumor tissues. We then found that the expression level of YAP protein in ccRCC tissues was significantly correlated with differentiation and clinical stage. In our immunohistochemistry study, the staining intensity of YAP was tightly linked with progression from well-differentiated to poorly differentiated tumors. Furthermore, YAP protein expression was correlated with clinical stage which may serve as an independent predictor for renal cancer. In the lower stages of ccRCC, there were low levels of expression of YAP, and higher expression of YAP was accompanied by higher stages of malignancy. In previous studies, YAP was found to play an important role in tumorigenesis and to be as an independent prognostic marker in HCC and ESCC $(24,25)$. Consistent with these research findings, the present study showed that the YAP gene is a tumor-specific gene, which is involved in the progression of ccRCC.

In previous studies, overexpression of YAP has been found in several human cancer cell types, and the oncogenic activity of YAP has been clearly demonstrated $(16,25,26)$. However, another study also reported that YAP may act as a tumor suppressor in certain breast carcinomas (27). The expression and activity of YAP in renal cancer cells are currently unknown. The present report showed that there was a significantly higher expression of YAP protein in ccRCC cell lines, 786-0 and ACHN, when compared with that in the HEK-293 cells. Specific shRNA lentiviral vectors targeting YAP were successfully constructed and transfected into 786-0 cells. We found that YAP mRNA and protein were effectively inhibited in the 786-0 cells. Furthermore, functional experiments were performed. The results indicated that silencing of YAP in 786-0 cells significantly inhibited cell proliferation, induced G1 phase cell cycle arrest and induced 786-0 cell apoptosis. Therefore, it is evident from the results that YAP plays an important role in the development of ccRCC.

In our initial experiment, we synthesized an siRNA targeting YAP and transfected it into 786-0 cells using Lipofectamine 2000, but it did not achieve the expected results; the effect of the suppression of the expression of the YAP gene was poor. Then, lentiviral vectors were engineered to knock down the YAP gene in 786-0 cells. All of the three YAP-shRNA interference groups were able to suppress the mRNA and protein expression levels of YAP. Further statistical analysis found that the lowest level of YAP mRNA expression in the 786-0 cells was in the shYAP-3 interference group, and the expression level of YAP protein in the shYAP-3 interference group cells was in accordance with the expression level of YAP mRNA. We chose lentiviral vectors for gene silencing due to their attractive properties, including high efficiency, persistent gene silencing and safety for humans $(28,29)$. Lentiviral vectors are a powerful tool for gene transfer into a broad range of cell types (30). Lentiviral vectors producing shRNAs have widespread use in the knockdown of gene expression both in vitro and in vivo.

Acting as downstream gene of the Hippo pathway, YAP is a transcription co-activator which interacts with the PPXYmotif-containing transcription factors, including TEAD, ErbB4, P73 and Runx2 (31,32). It has been reported that YAP and TEAD1 genetically interact to promote tissue growth (33). TEAD as a new component in the Hippo pathway plays an essential role in mediating the biological functions of YAP. In humans, there are four TEAD family members. YAP and TEAD1 bind to a common set of promoters in MCF10A cells (33). Furthermore, TEAD1 has been shown to be linked to the regulation of YAP in Drosophila and mammalian epithelial cell lines (34). A recent study has provided proof of principle that inhibiting TEAD-YAP interactions is a pharmacologically viable strategy against the YAP oncoprotein (35). TEAD has been demonstrated to be important for the growth-promoting function of YAP (36). The present study showed that silencing of YAP led to the decreased expression of TEAD1 and resulted in the growth inhibition of 786-0 cells. Thus, we speculated that the relevant mechanism involved the suppression of TEAD1 expression by the knockdown of YAP which consequently affects the expression of downstream cell growth factors and thus plays functional roles in the regulation of 786-0 cell proliferation. Yet this may not be the sole mechanism, and further research on the molecular functions of the YAP gene in renal cancer is warranted.

In conclusion, these findings demonstrated that YAP is an oncogene overexpressed in ccRCC, and the expression level of YAP protein is closely correlated with clinical stage and differentiation of ccRCC tissues. Moreover, suppression of YAP expression by YAP-shRNA lentiviral vectors reduced 786-0 cell growth and arrested the cell cycle at the G1 phase and also led to induced apoptosis. Therefore, YAP could be a potential novel therapeutic target for the treatment of ccRCC. 


\section{Acknowledgements}

The present study was supported by the Natural Science Foundation of China (grant no. 30972999) and the Nature Science Foundation of Chongqing (no. cqpc2012jja1698).

\section{References}

1. Ferlay J, Shin HR, Bray F, Forman D, Mathers C and Parkin DM Estimates of worldwide burden of cancer in 2008: GLOBOCAN 2008. Int J Cancer 127: 2893-2917, 2010.

2. Quivy A, Daste A, Harbaoui A, Duc S, Bernhard JC, GrossGoupil $\mathrm{M}$ and Ravaud A: Optimal management of renal cell carcinoma in the elderly: a review. Clin Interv Aging 8: 433-442, 2013.

3. Rathmell WK and Godley PA: Recent updates in renal cell carcinoma. Curr Opin Oncol 22: 250-256, 2010.

4. Vasudev NS, Selby PJ and Banks RE: Renal cancer biomarkers: the promise of personalized care. BMC Med 10: 112, 2012.

5. Edgar BA: From cell structure to transcription: Hippo forges a new path. Cell 124: 267-273, 2006.

6. Zhao B, Li L, Lei Q and Guan KL: The Hippo-YAP pathway in organ size control and tumorigenesis: an updated version. Genes Dev 24: 862-874, 2010

7. Pan D: The hippo signaling pathway in development and cancer. Dev Cell 19: 491-505, 2010

8. Zhao B, Wei X, Li W, et al: Inactivation of YAP oncoprotein by the Hippo pathway is involved in cell contact inhibition and tissue growth control. Genes Dev 21: 2747-2761, 2007.

9. Stanger BZ: Quit your YAPing: a new target for cancer therapy. Genes Dev 26: 1263-1267, 2012.

10. Zhang J, Ji JY, Yu M, et al: YAP-dependent induction of amphiregulin identifies a non-cell-autonomous component of the Hippo pathway. Nat Cell Biol 11: 1444-1450, 2009.

11. Bao Y, Hata Y, Ikeda M and Withanage K: Mammalian Hippo pathway: from development to cancer and beyond. J Biochem 149: 361-379, 2011.

12. Dong J, Feldmann G, Huang J, et al: Elucidation of a universal size-control mechanism in Drosophila and mammals. Cell 130: $1120-1133,2007$.

13. Zhao B, Tumaneng K and Guan KL: The Hippo pathway in organ size control, tissue regeneration and stem cell self-renewal. Nat Cell Biol 13: 877-883, 2011.

14. Harvey K and Tapon N: The Salvador-Warts-Hippo pathway an emerging tumour-suppressor network. Nat Rev Cancer 7: $182-191,2007$

15. Sawada A, Kiyonari H, Ukita K, Nishioka N, Imuta $Y$ and Sasaki H: Redundant roles of Teadl and Tead2 in notochord development and the regulation of cell proliferation and survival. Mol Cell Biol 28: 3177-3189, 2008.

16. Chan SW, Lim CJ, Chen L, Chong YF, Huang C, Song H and Hong W: The Hippo pathway in biological control and cancer development. J Cell Physiol 226: 928-939, 2011.

17. Lamar JM, Stern P, Liu H, Schindler JW, Jiang ZG and Hynes RO: The Hippo pathway target, YAP, promotes metastasis through its TEAD-interaction domain. Proc Natl Acad Sci USA 109: E2441-E2450, 2012.
18. Zender L, Spector MS, Xue W, et al: Identification and validation of oncogenes in liver cancer using an integrative oncogenomic approach. Cell 125: 1253-1267, 2006.

19. Li H, Wolfe A, Septer S, et al: Deregulation of Hippo kinase signalling in human hepatic malignancies. Liver Int 32: 38-47, 2012.

20. Steinhardt AA, Gayyed MF, Klein AP, et al: Expression of Yes-associated protein in common solid tumors. Hum Pathol 39: $1582-1589,2008$.

21. Zhang J, Smolen GA and Haber DA: Negative regulation of YAP by LATS1 underscores evolutionary conservation of the Drosophila Hippo pathway. Cancer Res 68: 2789-2794, 2008.

22. Zhao B, Li L, Wang L, Wang CY, Yu J and Guan KL: Cell detachment activates the Hippo pathway via cytoskeleton reorganization to induce anoikis. Genes Dev 26: 54-68, 2012.

23. Wang Y, Dong Q, Zhang Q, Li Z, Wang E and Qiu X: Overexpression of yes-associated protein contributes to progression and poor prognosis of non-small-cell lung cancer. Cancer Sci 101: 1279-1285, 2010

24. Xu MZ, Yao TJ, Lee NP, et al: Yes-associated protein is an independent prognostic marker in hepatocellular carcinoma. Cancer 115: 4576-4585, 2009.

25. Muramatsu T, Imoto I, Matsui T, et al: YAP is a candidate oncogene for esophageal squamous cell carcinoma. Carcinogenesis 32: 389-398, 2011.

26. Diep CH, Zucker KM, Hostetter G, et al: Down-regulation of Yes associated protein 1 expression reduces cell proliferation and clonogenicity of pancreatic cancer cells. PLoS One 7: e32783, 2012.

27. Tufail R, Jorda M, Zhao W, Reis I and Nawaz Z: Loss of Yes-associated protein (YAP) expression is associated with estrogen and progesterone receptors negativity in invasive breast carcinomas. Breast Cancer Res Treat 131: 743-750, 2012.

28. Sakuma T, Barry MA and Ikeda Y: Lentiviral vectors: basic to translational. Biochem J 443: 603-618, 2012.

29. Abbas-Terki T, Blanco-Bose W, Déglon N, Pralong W and Aebischer P: Lentiviral-mediated RNA interference. Hum Gene Ther 13: 2197-2201, 2002

30. Santamaria J, Khalfallah O, Sauty C, et al: Silencing of choline acetyltransferase expression by lentivirus-mediated RNA interference in cultured cells and in the adult rodent brain. J Neurosci Res 87: 532-544, 2009.

31. Strano S, Monti O, Pediconi N, et al: The transcriptional coactivator Yes-associated protein drives p73 gene-target specificity in response to DNA damage. Mol Cell 18: 447-459, 2005.

32. Li Z, Zhao B, Wang P, et al: Structural insights into the YAP and TEAD complex. Genes Dev 24: 235-240, 2010.

33. Zhao B, Ye X, Yu J, et al: TEAD mediates YAP-dependent gene induction and growth control. Genes Dev 22: 1962-1971, 2008.

34. Cao X, Pfaff SL and Gage FH: YAP regulates neural progenitor cell number via the TEA domain transcription factor. Genes Dev 22: 3320-3334, 2008.

35. Liu-Chittenden Y, Huang B, Shim JS, et al: Genetic and pharmacological disruption of the TEAD-YAP complex suppresses the oncogenic activity of YAP. Genes Dev 26: 1300-1305, 2012.

36. Ota M and Sasaki H: Mammalian Tead proteins regulate cell proliferation and contact inhibition as transcriptional mediators of Hippo signaling. Development 135: 4059-4069, 2008. 